\title{
pHluorin2: an enhanced, ratiometric, pH-sensitive green florescent protein
}

\author{
Matthew John Mahon
}

Department of Medicine, Harvard Medical School, Boston, USA.

E-mail: mahon@helix.mgh.harvard.edu

Received 7 April 2011; revised 9 May 2011; accepted 14 May 2011.

\begin{abstract}
Green florescent protein (GFP) variants that are sensitive to changes in $\mathrm{pH}$ are invaluable reagents for the analysis of protein dynamics associated with both endo- and exocytotic vesicular trafficking. Ratiometric pHluorin is a GFP variant that displays a bimodal excitation spectrum with peaks at 395 and $475 \mathrm{~nm}$ and an emission maximum at $509 \mathrm{~nm}$. Upon acidification, pHluorin excitation at $395 \mathrm{~nm}$ decreases with a corresponding increase in the excitation at $475 \mathrm{~nm}$. GFP2, a GFP variant that contains mammalianized codons and the folding enhancing mutation F64L, displays 8-fold higher florescence compared to pHluorin upon excitation at $395 \mathrm{~nm}$. Using GFP2 as a template, an enhanced ratiometric pHluorin (pHluorin2) construct was developed to contain fully mammalianized codons, the F64L mutation and ten of the thirteen pHluorin-specific mutations. As a result, $\mathrm{pH}$ luorin2 displays markedly higher florescence when compared to pHluorin while maintaining the ratiometric pH-sensitivity. Unlike native pHluorin, pHluorin2 expressed in the ligand-binding domain of the parathyroid hormone 1 receptor is readily detectable by confocal microscopy and displays a marked increase in florescence upon ligand-induced endocytosis to intracellular vesicles. Thus, pHluorin2 displays enhanced florescence while sustaining ratiometric $\mathrm{pH}-$ sensitivity, representing a significant improvement for this methodological approach.
\end{abstract}

Keywords: GFP; pHluorin; pH Sensing; Endocytosis

\section{INTRODUCTION}

The green florescent protein (GFP) from Aequorea victoria and its many engineered variants have revolutionized the analysis of protein dynamics [1]. Proteins of interest fused to GFP, typically from either the amino- or carboxy terminal ends, facilitates cellular localization from either fixed samples or for real-time analysis using spinning disk confocal microscopy. GFPs have also enabled the analysis of real-time, intracellular proteinprotein interactions using resonance energy transfer techniques, such as BRET (bioluminescence resonance energy transfer) and FRET (Forster resonance energy transfer) [2]. Notably, GFPs fluoresce autonomously and thus do not require either exogenous or endogenous co-factors, which is an important attribute when analyzing protein localization in various cellular compartments. Several reports demonstrate that the excitation/emission profiles of specific GFP variants are sensitive to $\mathrm{pH}$ changes [3-6].

Using a directed mutagenesis approach in E. coli, Rothman and coworkers [3] developed two, $\mathrm{pH}$-sensitive GFP variants, termed ecliptic- and ratiometric pHluorin, each containing clusters of mutations that direct $\mathrm{pH}$ sensitivity. Native GFP possesses a bimodal excitation profile with a major peak at $395 \mathrm{~nm}$ and a minor peak at $475 \mathrm{~nm}$ measured with an emission maximum at $509 \mathrm{~nm}$. Under acidic conditions ( $\mathrm{pH}<6$ ), ecliptic pHluorin is non-florescent, while shifting to a neutral environment enhances florescence at both peaks of excitation. Targeted expression of ecliptic pHluorin to acidic secretory vesicles allows for the real-time analysis of the exocytotic fusion of these vesicles to the plasma membrane where the $\mathrm{pH}$ becomes neutral, resulting in a corresponding increase in florescence [3]. A fluorescently enhanced variant of GFP, termed EGFP, was developed through the inclusion of the $\mathrm{F} 64 \mathrm{~L}$ and $\mathrm{S} 65 \mathrm{~T}$ mutations, changes that promote protein folding at $37^{\circ} \mathrm{C}$ and a shift in the major excitation peak to $475 \mathrm{~nm}$, respectively [7]. Sankarararayanan et al. [8] developed a superecliptic variant through incorporation of these same F64L and S65T mutations, changes that markedly enhance florescent output at the $475 \mathrm{~nm}$ excitation peak while maintaining $\mathrm{pH}$ sensitivity. The superecliptic pHluorin is ideal for analyzing exocytosis because florescence increases through progression of this process, i.e. acidic to neutral. However, using the same variant for the analysis of endocytosis would result in a loss of florescence over 
time, due to transit from a neutral environment (plasma membrane) to an acidic vesicle. The reduction of florescence could have two possible interpretations; the transit of tagged proteins to acidic vesicles or the proteolytic breakdown of these tagged proteins in lysosomes, leading to confounding analyses. Therefore, a $\mathrm{pH}$-sensor that displays an inverse relationship between $\mathrm{pH}$ and florescence output would be ideal for the analysis of endocytotic processes.

The second, ratiometric variant developed by Miesenbock et al. [3] displays the desirable characteristics for analysis of endocytosis. With increasing acidity, the ratiometric pHluorin displays a dose-dependent decrease in the excitation at $395 \mathrm{~nm}$ with a concomitant increase in the excitation at $475 \mathrm{~nm} \mathrm{[3].} \mathrm{These} \mathrm{characteristics}$ make ratiometric pHluorin an ideal reagent for monitoring cellular pathways that connect compartments that progress from neutral $\mathrm{pH}$ towards acidic ones, such as processes associated with endocytotic vesicular trafficking. Analysis of ligand-induced internalization of $\mathrm{G}$ protein coupled receptors (GPCRs) could benefit from this type of sensor. Analogous to the development of the superecliptic pHluorin [8], inclusion of mammalianized codons and the F64L mutation in the ratiometric pHluorin backbone markedly enhances florescence while maintaining the desired $\mathrm{pH}$-sensitivity, yielding a super-ratiometric variant called pHluorin2.

\section{MATERIALS AND METHODS}

\subsection{DNA Constructs and Cloning}

The ratiometric pHluorin construct was a generous gift from Dr. Rothman and the GFP2 construct was from Perkin-Elmer (Boston, MA). Using PCR directed by pfu polymerase (Agilent Technologies, Santa Clara, CA), both pHluorin and GFP2 were cloned into pcDNA3.1 (Invitrogen, Carlsbad, CA) as single, soluble proteins. Compared to native GFP, the GFP2 construct contains the F64L mutation and mammalianized codons (i.e. codon usage that is fully optimized for translation on mammalian ribosomes). Using site-directed, PCR-mediated mutagenesis, pHluorin-specific mutations were incorporated into the GFP2 construct, yielding the $\mathrm{pH}$ luorin2 construct, which contains the F64L mutation and mammalianized codons. Relative to GFP2, the pHluorin2-specific mutations include S147E, N149L, I161T, V163A, N164I, K166Q, I167V, R168H, S202H and L231H. Native pHluorin mutations not included in the pHluorin2 construct are Q80R, E132D and S175G. The pHluorin2 cDNA was then cloned in-frame within exon 2 between amino acids 95 and 96 of the human parathyroid hormone 1 receptor, yielding the hPTH1RpHluorin2 construct. Inclusion of EGFP within this domain does not interfere with ligand binding, internaliza- tion or signaling [9].

\subsection{Florescence Measurements}

Excitation scans were generated using a Deltascan dualwavelength fluorimeter from Photon Technology International (PTI; Birmingham, NJ) and the associated Felix software. Standard HEK293 cells were plated on $10 \mathrm{~cm}$ dishes and transiently transfected with $5 \mu \mathrm{g}$ each of the soluble forms of GFP2, pHluorin and pHluorin2 using Fugene HD (Roche, Indianapolis, IN). Forty-eight hours post transfection, cells were washed with Hank's Balanced Salt solution lacking calcium and magnesium ions and containing $2 \mathrm{mM}$ EDTA. Cells were lifted from the plates using the same solution after a 10 minute incubation at $37^{\circ} \mathrm{C}$ and placed in a quartz cuvette with a stir bar. Excitation scans were performed between wave- lengths $370 \mathrm{~nm}$ to $490 \mathrm{~nm}$ with the emission set at $510 \mathrm{~nm}$. For the $\mathrm{pH}$ clamp experiments, cells were resuspended in a buffer containing $140 \mathrm{mM}$ potassium chloride, $10 \mathrm{mM}$ sodium phosphate with molar ratios of mono- and dibasic forms appropriate for the given $\mathrm{pH}$ and $30 \mu \mathrm{M}$ nigericin (EMD Biosciences, Gibbstown, NJ). For the florescence plate reader studies, black, 96-well plates (Perkin-Elmer, Boston, MA) were loaded with 25,000 cells per well from the transfections described above and analyzed with dual excitation at $405 \mathrm{~nm} / 8 \mathrm{~nm}$ and $485 \mathrm{~nm} /$ $25 \mathrm{~nm}$ with a $535 \mathrm{~nm} / 25 \mathrm{~nm}$ emission filter in an EnVision plate reader (Perkin-Elmer, Boston, MA). The narrow bandwidth of the $405 \mathrm{~nm}$ filter of $8 \mathrm{~nm}$ reduces the relative florescence compared to the larger bandwidth of the $485 \mathrm{~nm}$ filter. Despite this effect, the 405/485 nm ratios are still responsive to changes in intracellular $\mathrm{pH}$.

For the hPTH1R endocytosis studies, HEK293 cells were sparsely plated into culture slides and transiently transfected with $100 \mathrm{ng}$ of the hPTH1R-pHluorin2 construct using Fugene HD. Forty-eight hours post-transfection, cells were treated with either vehicle (acetic acid) or $100 \mathrm{nM}$ parathyroid hormone containing amino acids 1 to 34 for 20 minutes. Cells were fixed with $3.7 \%$ paraformaldehyde and analyzed by confocal microscopy using a Radiance 2100 confocal microscope and the associated LaserSharp 2000 software (Bio-Rad Laboratories, Inc., Hercules, CA).

\section{RESULTS AND DISCUSSION}

The excitation spectrum for native GFP is bimodal with a major peak at $395 \mathrm{~nm}$ and a minor peak at $475 \mathrm{~nm}$ with an emission maximum at $509 \mathrm{~nm}$. GFP2, a variant developed for the use in BRET applications, contains fully mammalianized codons and the florescence enhancing mutation F64L. GFP2 and pHluorin display the same native GFP-specific excitation profile. However, the relative florescence of the $395 \mathrm{~nm}$ excitation peak of 
GFP2 is approximately 8-fold higher than pHluorin (Figure 1(a)). Several possible reasons regarding this marked difference in florescence exist. First, the several pHluorin-specific mutations, which impart the $\mathrm{pH}$-sensitivity, may hamper the florescent output. However, this effect seems unlikely because pHluorin was selected based on its florescence characteristics when expressed in bacteria [3]. This mutagenesis process, however, raises the possibility that the selected codons favor bacterial expression over those codons preferred by mammalian ribosomes. This possible effect is further compounded by the fact that pHluorin was developed from gene sequences consistent with the original jellyfish GFP cDNA. In contrast, the synthetic GFP2 construct contains fully mammalianized codons. Lastly, unlike pHluorin, all of the enhanced variants of GFP contain a leucine for phenylalanine substitution at amino acid 64 (F64L), including GFP2, enhanced cyan florescent protein (ECFP), enhanced green florescent protein (EGFP) and enhanced yellow florescent protein (EYFP) [7]. It is thought that the F64L mutation markedly enhances florescence by promoting proper protein folding at $37^{\circ} \mathrm{C}$ [10].

Using PCR-mediated mutagenesis and the GFP2 cDNA as a template, an enhanced version of ratiometric pHluorin (pHluorin2) was developed. PHluorin2 contains the F64L mutation and ten out of the thirteen pHluorin-specific mutations (Table 1). It is not known if the remaining mutations (Q80R, E132D and S175G) will have additional effects, but at this stage they have been determined to be unnecessary. Furthermore, the pHluorin2 cDNA contains fully mammalianized codons. As a result of these changes, the florescence output of pHluorin2 is 8-fold higher than native pHluorin when excited at $395 \mathrm{~nm}$ to levels that are comparable to GFP2 (Figure 1(a)). Importantly, $\mathrm{pH}$ clamp experiments using nigeri cin clearly demonstrate that the elements included to enhance pHluorin2 florescence do not affect the ratiometric, $\mathrm{pH}$-sensitive characteristics attributed to the original pHluorin-specific mutations (Figure 1(b)). Compared to the pHluorin expressed in bacteria [3], the $\mathrm{pH}$-dependent difference in the florescence maxima at $475 \mathrm{~nm}$ also appears greater for pHluorin2 when expressed in mammalian cells (Figure 1(b)). The markedly enhanced florescent output while maintaining $\mathrm{pH}$-sensitivity makes pHluorin2 a valuable reagent for the analysis of changes in intracellular $\mathrm{pH}$.

Similar to 2', 7'-bis-(2-carboxyethyl)-5-(and-6)-carboxyfluorescein (BCECF), pHluorin2 can monitor changes in intracellular $\mathrm{pH}$ using a standard plate florimeter. Consistent with the data described above, the relative florescence of the pHluorin2 is $\sim 8$-fold higher than pHluorin when analyzed with a plate florimeter (Figures 2(A) and 2(B)). Dual excitation at $405 \mathrm{~nm}$ and $485 \mathrm{~nm}$ yields $405 \mathrm{~nm} / 485 \mathrm{~nm}$ ratios that decrease in res-

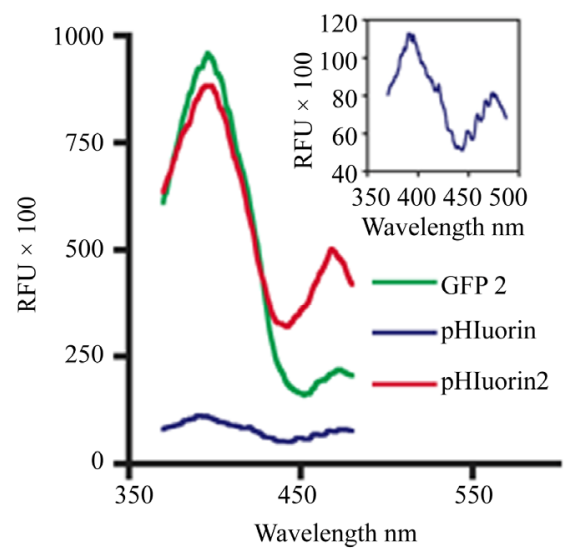

(a)

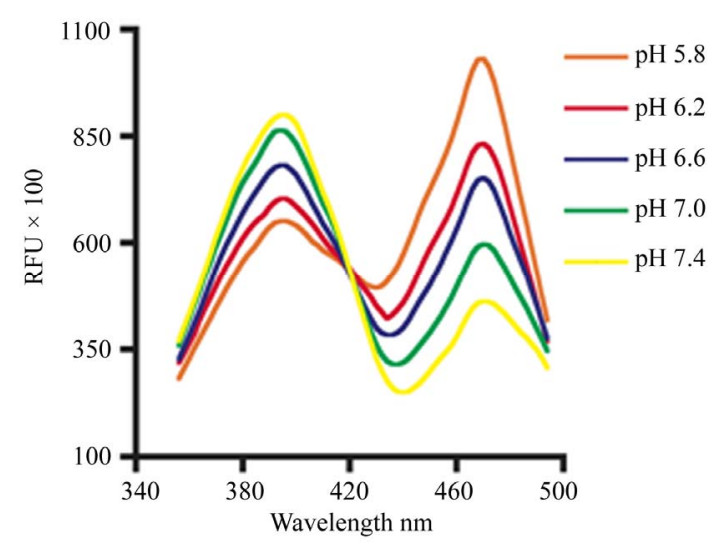

(b)

Figure 1. Compared to pHluorin, pHluorin2 displays a marked increase in florescence while maintaining ratiometric, pH-sensing. (a) HEK293 cells were transiently transfected with the cytoplasmic forms of GFP2, pHluorin and pHluorin2. Relative florescence units (RFU) of excitation scans between $370 \mathrm{~nm}$ and $490 \mathrm{~nm}$ with an emission set at $510 \mathrm{~nm}$ for GFP2 (green line), pHluorin (blue line and inset graph) and pHluorin2 (red line) are shown; (b) Excitation scans of cytoplasmic pHluorin2 expressed in HEK293 cells clamped at the indicated pH using $\mathrm{K}^{+}$ions and nigericin are shown. Data are representative of three independent experiments.

ponse to lower intracellular $\mathrm{pH}$ for both pHluorin and pHluorin2. Notably, these ratios are similar for both proteins, illustrating the benefit of ratiometric analysis, which normalizes expression levels of the florescent protein. Using the nigericin $\mathrm{pH}$ clamp methodology, a $\mathrm{pH}$ versus the $405 \mathrm{~nm} / 485 \mathrm{~nm}$ ratio standard curve was generated (data not shown). Applying this standard curve, cytoplasmic pHluorin2 is capable of monitoring nigercin-induced cellular acidification over time using a plate florimeter (Figure 2 (c)).

The parathyroid hormone 1 receptor (PTH1R), a primary regulator of mineral ion homeostasis, is a class $b \mathrm{G}$ 
Table 1. Amino acid comparisons between GFP2, pHluorin and pHluorin2*

\begin{tabular}{|c|c|}
\hline & 1 \\
\hline GFP2 & MSKGEELFTG VVPILVELDG \\
\hline pHluorin & MSKGEELFTG VVPILVELDG \\
\hline pHluorin2 & $\begin{array}{l}\text { MSKGEELFTG VVPILVELDG } \\
21\end{array}$ \\
\hline GFP2 & DVNGHKFSVS GEGEGDATYG \\
\hline pHluorin & DVNGHKFSVS GEGEGDATYG \\
\hline pHluorin2 & $\begin{array}{l}\text { DVNGHKFSVS GEGEGDATYG } \\
41\end{array}$ \\
\hline GFP2 & KLTLKFICTT GKLPVPWPTL \\
\hline pHluorin & KLTLKFICTT GKLPVPWPTL \\
\hline pHluorin2 & $\begin{array}{l}\text { KLTLKFICTT GKLPVPWPTL } \\
61\end{array}$ \\
\hline GFP2 & VTTLSYGVQC FSRYPDHMKQ \\
\hline pHluorin & VTTFSYGVQC FSRYPDHMKR \\
\hline pHluorin2 & $\begin{array}{l}\text { VTTLSYGVQC FSRYPDHMKQ } \\
81\end{array}$ \\
\hline GFP2 & HDFFKSAMPE GYVQERTIFF \\
\hline pHluorin & HDFFKSAMPE GYVQERTIFF \\
\hline pHluorin2 & $\begin{array}{l}\text { HDFFKSAMPE GYVQERTIFF } \\
101\end{array}$ \\
\hline GFP2 & KDDGNYKTRA EVKFEGDTLV \\
\hline pHluorin & KDDGNYKTRA EVKFEGDTLV \\
\hline pHluorin2 & $\begin{array}{l}\text { KDDGNYKTRA EVKFEGDTLV } \\
121\end{array}$ \\
\hline GFP2 & NRIELKGIDF KEDGNILGHK \\
\hline pHluorin & NRIELKGIDF KDDGNILGHK \\
\hline pHluorin2 & $\begin{array}{l}\text { NRIELKGIDF KEDGNILGHK } \\
141\end{array}$ \\
\hline GFP2 & LEYNYNSHNV YIMADKQKNG \\
\hline pHluorin & LEYNYNEHLV YIMADKQKNG \\
\hline pHluorin2 & $\begin{array}{l}\text { LEYNYNEHLV YIMADKQKNG } \\
161\end{array}$ \\
\hline GFP2 & IKVNFKIRHN IEDGSVQLAD \\
\hline pHluorin & TKAIFQVHHN IEDGGVQLAD \\
\hline pHluorin2 & $\begin{array}{l}\text { TKAIFQVHHN IEDGGVQLAD } \\
181\end{array}$ \\
\hline GFP2 & HYQQNTPIGD GPVLLPDNHY \\
\hline pHluorin & HYQQNTPIGD GPVLLPDNHY \\
\hline pHluorin2 & $\begin{array}{l}\text { HYQQNTPIGD GPVLLPDNHY } \\
201\end{array}$ \\
\hline GFP2 & LSTQSALSKD PNEKRDHMVL \\
\hline pHluorin & LHTQSALSKD PNEKRDHMVL \\
\hline pHluorin2 & $\begin{array}{l}\text { LHTQSALSKD PNEKRDHMVL } \\
221\end{array}$ \\
\hline GFP2 & LEFVTAAGIT LGMDELYK* \\
\hline pHluorin & LEFVTAAGIT HGMDELYK* \\
\hline pHluorin2 & LEFVTAAGIT HGMDELYK* \\
\hline
\end{tabular}

*Amino acids in red are specific to GFP2 and pHluorin2. Amino acids in green are pHluorin-specific mutations incorporated into the pHluorin2 construct. The protein folding enhancing mutation, F64L, is boxed.

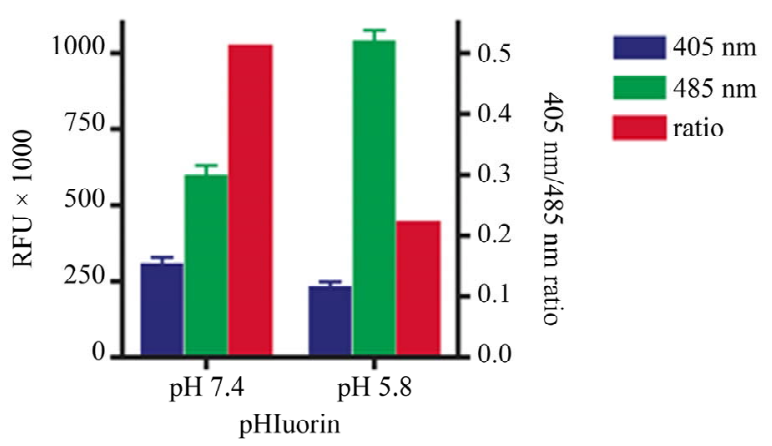

(a)

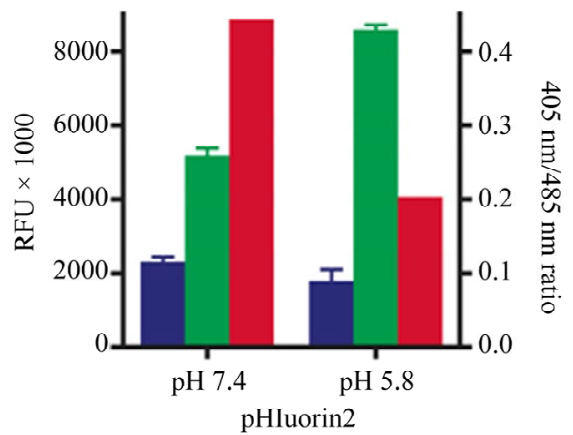

(b)

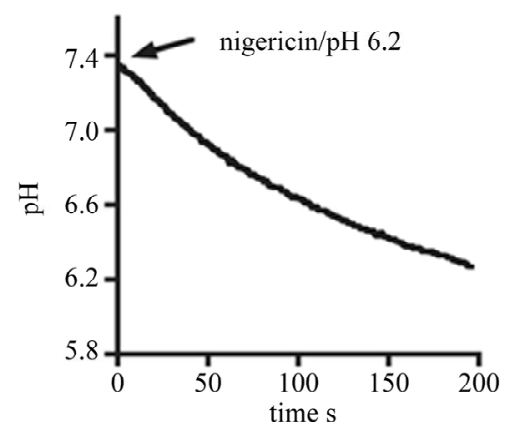

(c)

Figure 2. Monitoring intracellular $\mathrm{pH}$ using pHluorin2 and a plate florimeterr. Cytoplasmic pHluorin (a) or pHluorin2 (b), expressed in HEK293 cells clamped at either $\mathrm{pH} 7.4$ or 5.8 with nigericin, as indicated, are analyzed in a plate florimeter by dual excitation at $405 \mathrm{~nm}$ (blue bars) and $485 \mathrm{~nm}$ (green bars) with an emission filter of $535 \mathrm{~nm}$. Ratios of $405 \mathrm{~nm} /$ $485 \mathrm{~nm}$ (red bars) are shown on the right Y-axis. (c) Nigericin-induced cellular acidification of HEK293 cells expressing pHluorin2 in extracellular buffer set at $\mathrm{pH} 6.2$ over time in seconds is shown. Data are representative of three independent experiments.

protein coupled receptor (GPCR) that binds both parathyroid hormone $(\mathrm{PTH})$ and parathyroid hormone related protein (PTHrP). Recent reports demonstrate that PTH1R endocytosis plays an important role in the differential cellular responses mediated by these two prominent hormones [11]. Thus, development of a high-throughput method capable of analyzing PTH1R endocytosis, or any GPCR for that matter, would greatly benefit this field of research. When EGFP is cloned in-frame within the 
second exon of the PTH1R, which is located in the extracellular ligand-binding domain, the receptor functions normally [9]. However, EGFP florescence expressed as a chimera with the PTH1R is lower when compared to EGFP expressed alone (unpublished observations). Consistent with this phenomenon, pHluorin expressed in this same domain of the PTH1R yields florescence that is marginally detectable above background when analyzed by confocal microscopy (data not shown). These initial observations prompted the development of pHluorin2. As a result of the enhanced florescence, pHluorin 2 cloned within this same domain of the PTH1R generates a receptor that is readily detected by confocal microscopy (Figure 3(a)). Notably, ligand-induced endocytosis results in the formation of PTH1R-containing vesicles that are markedly brighter than when the receptor is on the cell surface, suggesting movement into an acidic vesicular environment (Figure 3(b)).

\section{CONCLUSIONS}

Since the original discovery and cloning of the wild-type GFP, several variants have been engineered with the goal to improve the utility of this protein for the analysis of protein dynamics. Development of the pHluorins by Rothman and co-workers [3] represents a seminal work in the achievement of these goals, especially as it relates to processes associated with compartmental changes in $\mathrm{pH}$, such as exo- and endocytosis. Two straightforward modifications of the original ratiometric pHluorin were included in the development of pHluorin2. First, inclusion of the F64L mutation was desirable because this modification enhances florescence without changing the spectral characteristics. This is in contrast to the S65T mutation specific to EGFP, which markedly enhances florescence but does so through shifting the major excitation peak from $395 \mathrm{~nm}$ to $475 \mathrm{~nm}$ [7]. Second, use of fully mammalianized codons assures that codon biases are not impeding full expression, especially when the original pHluorin construct was developed in bacteria.

With respect to ligand-induced receptor endocytosis, such as that demonstrated for GPCRs, high throughput methods monitoring transit from the neutral extracellular plasma membrane to acidic vesicles would certainly benefit from the use of $\mathrm{pH}$-sensitive GFPs. For example, the enhanced yellow florescent protein (EYFP) displays a marked decrease in florescence upon acidification [4]. Similar to ecliptic pHluorin, loss of florescence could signify receptor localization to acidic vesicles; however, as discussed earlier, a potential drawback is that one cannot rule receptor degradation as a possible explanation for decreased florescence. Therefore, use of the ratiometric forms of pHluorin is more desirable because the ligand-induced receptor transit from the cell surface

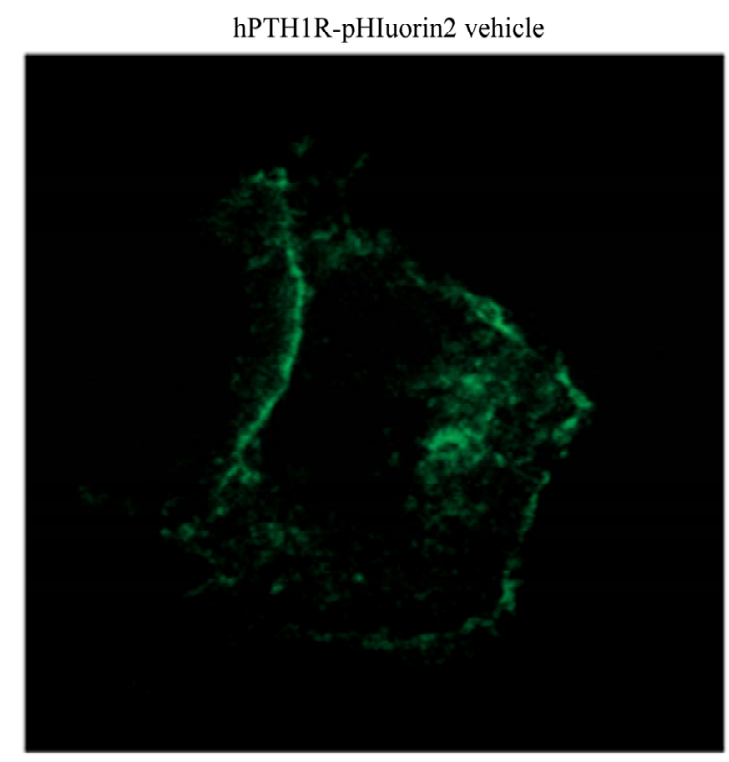

(a)

$100 \mathrm{nM}$ PTH

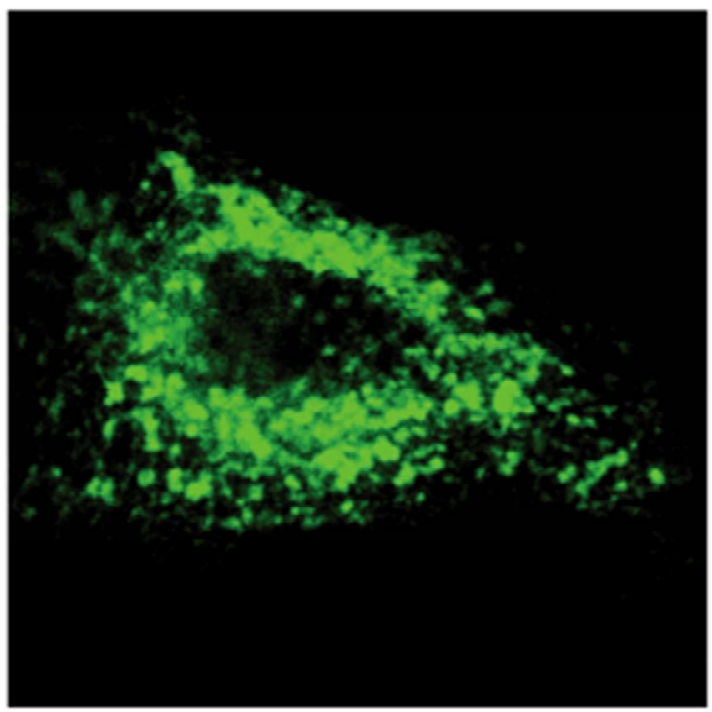

(b)

Figure 3. Enhanced florescence in the FITC-channel upon ligand-induced endocytosis of hPTH1R-pHluorin2. HEK293 cells expressing hPTH1R-pHluorin2 were treated with either vehicle (a) or $100 \mathrm{nM}$ PTH(1-34) (b) Representative confocal images using the FITC-channel with identical laser power and gain are shown.

to the endocytic vesicles is marked by an increase in florescence using the common FITC-type filter sets. Furthermore, the enhanced florescence displayed by pHluorin2 increases sensitivity and thus reduces the need to grossly over-express the receptor chimera of interest. Combined, the enhanced sensitivity and expression of pHluorin2 will increase the applicability and effectiveness of this construct. 


\section{ACKNOWLEDGEMENTS}

I thank Dr. James Rothman for the ratiometric pHluorin construct. This work is supported by the NIDDK Program Project Grant 5P01DK011794-42 of Dr. John Potts. This paper is subject to the NIH Public Access Policy.

\section{REFERENCES}

[1] Shaner, N.C., Patterson, G.H. and Davidson, M.W. (2007) Advances in fluorescent protein technology. Journal of Cell Science, 120, 4247-4260. doi:10.1242/jcs.005801

[2] Ciruela, F. (2008) Fluorescence-based methods in the study of protein-protein interactions in living cells. Current Opinion in Biotechnology, 19, 338-343. doi:10.1016/j.copbio.2008.06.003

[3] Miesenbock, G., De Angelis, D.A. and Rothman, J.E. (1998) Visualizing secretion and synaptic transmission with $\mathrm{pH}$-sensitive green fluorescent proteins. Nature, 394, 192-195. doi: $10.1038 / 28190$

[4] Llopis, J., McCaffery, J.M., Miyawaki, A., et al. (1998) Measurement of cytosolic, mitochondrial, and Golgi $\mathrm{pH}$ in single living cells with green fluorescent proteins. Proceedings of the National Academy of Sciences USA, 95, 6803-6808. doi:10.1073/pnas.95.12.6803

[5] Awaji, T., Hirasawa, A., Shirakawa, H., et al. (2001) Novel green fluorescent protein-based ratiometric indi- cators for monitoring $\mathrm{pH}$ in defined intracellular microdomains. Biochemical Biophysical Research Communications, 289, 457-462. doi:10.1006/bbrc.2001.6004

[6] Bizzarri, R., Arcangeli, C., Arosio, D., et al. (2006) Development of a novel GFP-based ratiometric excitation and emission $\mathrm{pH}$ indicator for intracellular studies. Biophysics Journal, 90, 3300-3314. doi:10.1529/biophysj.105.074708

[7] Heim, R., Cubitt, A.B. and Tsien, R.Y. (1995) Improved green fluorescence. Nature, 373, 663-664. doi: $10.1038 / 373663 b 0$

[8] Sankaranarayanan, S., De Angelis, D., Rothman, J.E., et al. (2000) The use of pHluorins for optical measurements of presynaptic activity. Biophys Journal, 79, 2199-2208. doi:10.1016/S0006-3495(00)76468-X

[9] Tawfeek, H.A., Qian, F. and Abou-Samra, A.B. (2002) Phosphorylation of the receptor for PTH and PTHrP is required for internalization and regulates receptor signaling. Molecular Endocrinology, 16, 1-13. doi: $10.1210 /$ me.16.1.1

[10] Thastrup O.T.S., et al. (1995) Fluorescent proteins. US patent number: 7314915 .

[11] Ferrandon, S., Feinstein, T.N., Castro, M., et al. (2009) Sustained cyclic AMP production by parathyroid hormone receptor endocytosis. Nature Chemical Biology, 5, 734-742. doi:10.1038/nchembio.206 